\title{
Pan Digital Gangrene: A Rare Phenomenon in Scrub Typhus
}

\author{
${ }^{1}$ Puneet Rijhwani, ${ }^{2}$ Abhishek Charan, ${ }^{3} \mathrm{CM}$ Agrawal, ${ }^{4}$ Manish R Pahadia, ${ }^{5}$ Shubham Gupta, ${ }^{6}$ Haritej Anand
}

\begin{abstract}
Introduction: Scrub typhus is an acute, febrile zoonosis, caused by an obligate intracellular bacterium Orientia tsutsugamushi. The disease is of greatest public health importance in rural areas of Asia and in Western Pacific Islands. The clinical manifestations of the disease range from subclinical disease to an organ failure. The various complications known with this disease are jaundice, renal failure, pneumonitis, acute respiratory distress syndrome, septic shock, myocarditis, vasculitis, and meningoencephalitis. The complications of scrub typhus usually develop after the first week of illness.
\end{abstract}

Case report: We report a case of a 70-year-old female, farmer by occupation, who presented with acute febrile illness and was diagnosed as scrub typhus. She subsequently developed vasculitis, which resulted in pan digital gangrene. After thorough workup for digital gangrene, it was established that scrub typhus is the cause of gangrene in this patient.

Conclusion: Thus, our inference is that vasculitis might be seen in very few cases of scrub typhus and it may progress up to gangrene of digits. Only one such case has been reported, thus making it a very rare phenomenon.

Keywords: Digital gangrene, Scrub typhus, Vasculitis.

How to cite this article: Rijhwani P, Charan A, Agrawal CM, Pahadia MR, Gupta S, Anand H. Pan Digital Gangrene: A Rare Phenomenon in Scrub Typhus. J Mahatma Gandhi Univ Med Sci Tech 2017;2(1):35-37.

\section{Source of support: Nil}

\section{Conflict of interest: None}

\section{INTRODUCTION}

Scrub typhus, caused by Orientia (formerly Rickettsia) tsutsugamushi, is an acute infectious disease of variable severity that is transmitted to humans by an arthropod vector of the Trombiculidae family. "Tsutsuga" means small and dangerous, and "mushi" means insect or mite. It affects people of all ages including children. Humans

\footnotetext{
${ }^{1}$ Professor, ${ }^{2,6}$ Postgraduate Resident (2nd year), ${ }^{3}$ Associate Professor, ${ }^{4}$ Assistant Professor, ${ }^{5}$ Postgraduate Resident (1st year)

${ }^{1-6}$ Department of Medicine, Mahatma Gandhi Medical College \& Hospital, Jaipur, Rajasthan, India

Corresponding Author: Puneet Rijhwani, Professor, Department of Medicine, Mahatma Gandhi Medical College \& Hospital Jaipur, Rajasthan, India, e-mail: puneet284@rediffmail.com
}

are accidental hosts in this zoonotic disease. While scrub typhus is confined geographically to the Asia-Pacific region, a billion people are at risk and nearly a million cases are reported every year. ${ }^{1}$ Mite can serve as both the vector and the reservoir.

It is endemic to a part of world known as "tsutsugamushi triangle," which extends from northern Japan and eastern Russia in the north to northern Australia in the south and to Pakistan and Afghanistan in the west. ${ }^{2}$ Scrub typhus is often acquired during occupational or agricultural exposures ${ }^{3}$ because active rice fields are an important reservoir for transmission. ${ }^{4}$

The incubation period may range from 5 to 21 days. The common symptoms are fever, chills, headache, myalgia, dry cough, lymphadenopathy, and gastrointestinal disturbances. Scrub typhus can affect skin, lung, heart, and central nervous system with the potential of causing serious life-threatening complications. The clinical and laboratory features are nonspecific in scrub typhus. The eschar is the single most useful diagnostic clue and is pathognomonic for O. tsutsugamushi, but is seen in less than $10 \%$ of cases in the Indian subcontinent.

\section{CASE REPORT}

A 70-year-old female, farmer by occupation, presented to our emergency department with complaints of highgrade fever associated with chills for 12 days. She had shortness of breath with dry cough for 3 days and from last 2 days she developed altered sensorium and then she was referred to our hospital from district hospital.

Arterial blood gas test was done and showed respiratory alkalosis. Patient was admitted in the intensive care unit and was kept on ventilator. Intravenous fluids with broad spectrum antibiotics were initiated and routine investigations along with scrub and dengue serology were sent.

On second day, doxycycline $100 \mathrm{mg}$ bd with azithromycin $500 \mathrm{mg}$ bd was started, as scrub typhus was confirmed. Patient developed blackish discoloration of digits of upper limb, which was later followed in lower limb also. Discoloration was progressive and eventually it turned into dry gangrene (Tables 1 and 2).

Mild calcification with no narrowing in bilateral axillary and brachial artery was seen in arterial Doppler study. However, there was multiple wall calcification and 


\begin{tabular}{ll}
\hline \multicolumn{2}{c}{ Table 1: Complete blood count } \\
\hline Bilirubin total & $0.80(0.20-1.30 \mathrm{mg} \%)$ \\
Bilirubin direct & $0.20(0.10-1.20 \mathrm{mg} \%)$ \\
SGOT (AST) & $135(15-46 \mathrm{U} / \mathrm{L})$ \\
SGPT (ALT) & $17(13.00-69.00 \mathrm{U} / \mathrm{L})$ \\
Alkaline phosphatase & $450(38.00-126.00 \mathrm{U} / \mathrm{L})$ \\
Urea & $58(15-45 \mathrm{mg} \%)$ \\
Creatinine & $0.9(0.52-1.25 \mathrm{mg} \%)$ \\
Uric acid & $7.5(2.5-6.2 \mathrm{mg} \%)$ \\
Serum sodium & $138.00(137-145 \mathrm{mmol} / \mathrm{L})$ \\
Serum potassium & $4.5(3.50-5.10 \mathrm{mmol} / \mathrm{L})$ \\
Serum chloride & $108(98-107 \mathrm{mmol} / \mathrm{L})$ \\
\hline SGOT: Serum glutamic oxaloacetic transaminase; SGPT: Serum \\
glutamic pyruvic transaminase; AST: Aspartate aminotransferase; \\
ALT: Alanine aminotransferase
\end{tabular}

narrowing in distal end of bilateral and ulnar artery. Ultrasonography was suggestive of distended gall bladder with echogenic sludge. Computed tomography brain, electrocardiogram, and echocardiogram were normal.

Autoimmune workup for vasculitis like antinuclear antibodies, antiphospholipid antibodies, C-antineutrophil cytoplasmic antibodies (ANCA), P-ANCA, anticentromere antibody were done and came out to be negative (Table 3).

However, C-reactive protein and rheumatoid arthritis (RA) factor were positive. But she had no clinical manifestation of RA and any other connective tissue disorder.

\section{DISCUSSION}

Scrub typhus is widespread in Indian subcontinent. With the involvement of multiple organs, severe complications may develop, which could make it a fatal disease.

Endothelial cells and macrophages are the main target cells for O. tsutsugamushi. It disseminates into multiple organs through endothelial cells via hematogenous and lymphatogenous routes and predominantly locates in the macrophages of the liver and spleen. ${ }^{5}$ The bacteria then cause focal or systemic vasculitis and perivasculitis in multiple organs, with various complications. Complications are seen in those patients who are left untreated in their
Table 2: Workup for fever

\begin{tabular}{ll}
\hline WBC & 9.35 \\
RBC & 4.27 \\
HGB & 11.1 \\
HCT & 31.5 \\
PLT & $1 \mathrm{Lac}$ \\
NEUT & $75.8 \%$ \\
LYMPH & $20.4 \%$ \\
ESR & $13 \mathrm{~mm}$ in $1^{\text {st }} \mathrm{hr}$ \\
\hline
\end{tabular}

Table 3: Autoimmune workup for fever

\begin{tabular}{ll}
\hline Malaria & Negative \\
Dengue Serology & Negative \\
Leptosira Serology & Negative \\
Anti HCV & Negative \\
HbsAg & Negative \\
HIV & Negative \\
\hline
\end{tabular}

first week of illness. The various complications known to occur with this disease are acute renal failure, acute hepatic failure, interstitial pneumonitis, acute respiratory distress syndrome, septic shock, myocarditis, pericarditis, meningoencephalitis, and also acute hearing loss. ${ }^{6,7}$

These complications are the result of endothelial damage and this forms the basis for systemic vasculitis. Sometimes, vaso-occlusion due to venous thrombosis may also lead to development of vasculitis.

Digital gangrene involving all digits of four limbs is a sign of systemic disease, such as infections like syphilis, leprosy, endocarditis, viral (hepatitis B, hepatitis C, human immunodeficiency virus), fungal, and parasites (Fig. 1). With risk factors, such as hypertension, diabetes, dyslipidemia, atherosclerosis has become the leading causes of peripheral arterial disease. Vasculitis and thrombophilic states should be ruled out in all cases of digital gangrene.

In primary systemic vasculitis, medium-sized vessel are commonly involved, such as polyarteritis nodosa, which is associated with hepatitis B, Wegener's granulomatosis, Churg-Strauss syndrome. Though uncommon, large-size vessel vasculitis can also lead to digital gangrene, such as giant cell arteritis and Takayasu arteritis. Digital ischemia is very commonly associated with
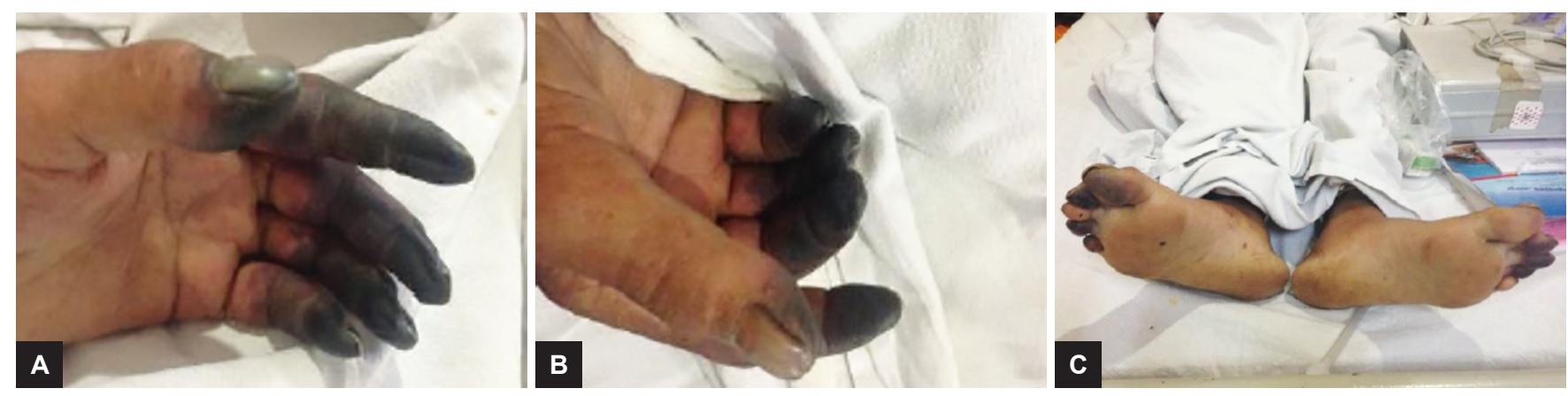

Figs $1 \mathrm{~A}$ to $\mathrm{C}$ : Dry gangrene involving all digits of $(A)$ right hand; $(B)$ left hand; and $(C)$ both foot 


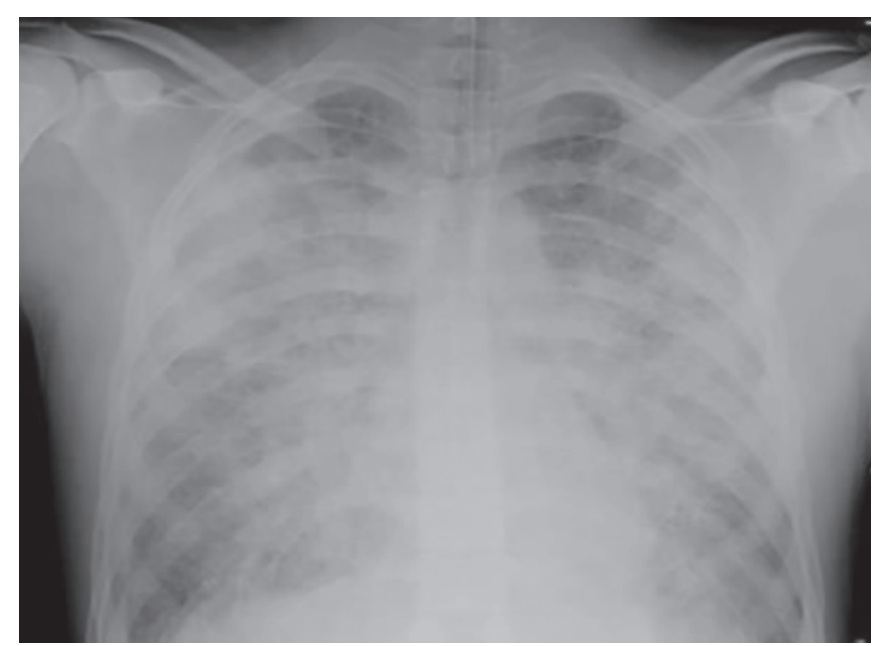

Fig. 2: Chest $X$ ray shows acute respiratory distress syndrome

systemic lupus erythematosus. Similarly, RA, scleroderma, antiphospholipid syndrome, Raynaud's phenomenon are well-known causes of vasculitis digital ischemia and should be kept in mind. Behçet disease is also associated with digital gangrene (Fig. 2).

All possible causes whether infective or noninfective were ruled out. And, eventually, we reached out to a conclusion that scrub typhus has led to pan digital gangrene in this patient.

\section{CONCLUSION}

Scrub typhus is a reemergent zoonosis disease in the Indian subcontinent. Due to its pathophysiology which involves endothelial dysfunction of small blood vessels it is a multiorgan disease. Vasculitis is one of the dreaded complications of scrub typhus, which can lead to digital ischemia and then gangrene.

Proper evaluation and early and prompt treatment can lead to effective control of its complication. While going through the literature, we found out that not many cases have been reported, so while dealing with pan digital gangrene, scrub typhus should be kept in mind.

\section{REFERENCES}

1. Watt G, Parola P. Scrub typhus and tropical rickettsioses. Curr Opin Infect Dis 2003 Oct;16(5):429-436.

2. http://emedicine.medscape.com/article/971797-overview\#a3.

3. Watt G, Walker DH. Scrub typhus. In: Guerrant RL, Walker $\mathrm{DH}$, Weller PF, editors. Tropical infectious diseases principles, pathogens and practice. 2nd ed. Philadelphia (PA): Elsevier Churchill Livingstone; 2006. Vol. 1. Chapter 52.

4. Berman SJ, Kundim WD. Scrub typhus in South Vietnam: a study of 87 cases. Ann Intern Med 1973 Jul;79(1):26-30.

5. Moron CG, Popov VL, Feng HM, Wear D, Walker DH. Identification of the target cells of Orientia tsutsugamushi in human cases of scrub typhus. Mod Pathol 2001 Aug;14(8): 752-759.

6. Cracco C, Delafosse C, Baril L, Lefort Y, Morelot C, Derenne JP, Bricaire F, Similowski T. Multiple organ failure complicating probable scrub typhus. Clin Infect Dis 2000 Jul;31(1):191-192.

7. Premaratna R, Chandrasena TG, Dassayake AS, Loftis AD, Dasch GA, de Silva HJ. Acute hearing loss due to scrub typhus: a forgotten complication of a reemerging disease. Clin Infect Dis 2006 Jan 1;42(1):e6-e8. 